\title{
The Arabidopsis PAD4 Lipase-Like Domain Is Sufficient for Resistance to Green Peach Aphid
}

\author{
Joram A. Dongus, ${ }^{1}$ Deepak D. Bhandari, ${ }^{1}$ Monika Patel, ${ }^{2}$ Lani Archer, ${ }^{2}$ Lucas Dijkgraaf, ${ }^{1,3}$ \\ Laurent Deslandes, ${ }^{4}$ Jyoti Shah, ${ }^{2}$ and Jane E. Parker ${ }^{1, \dagger}$ \\ ${ }^{1}$ Department of Plant-Microbe Interactions, Max Planck Institute for Plant Breeding Research, Carl-von-Linné-weg 10, 50829 \\ Cologne, Germany \\ ${ }^{2}$ Department of Biological Sciences, University of North Texas; 1511 West Sycamore, Denton, TX 76201, U.S.A. \\ ${ }^{3}$ Plant-Microbe Interactions - Utrecht University, Padualaan 8; $3584 \mathrm{CH}$ Utrecht, Netherlands \\ ${ }^{4}$ LIPM, Université de Toulouse, INRA, CNRS, Castanet-Tolosan, France
}

Accepted 6 November 2019.

\begin{abstract}
Plants have evolved mechanisms to protect themselves against pathogenic microbes and insect pests. In Arabidopsis, the immune regulator PAD4 functions with its cognate partner EDS1 to limit pathogen growth. PAD4, independently of EDS1, reduces infestation by green peach aphid (GPA). How PAD4 regulates these defense outputs is unclear. By expressing the $\mathrm{N}$ terminal PAD4 lipase-like domain (PAD4 ${ }^{\mathbf{L L D}}$ ) without its Cterminal EDS1-PAD4 (EP) domain, we interrogated PAD4 functions in plant defense. Here, we show that transgenic expression of PAD4 ${ }^{\mathrm{LLD}}$ in Arabidopsis is sufficient for limiting GPA infestation but not for conferring basal and effectortriggered pathogen immunity. This suggests that the $C$ terminal PAD4 EP domain is necessary for EDS1-dependent immune functions but is dispensable for aphid resistance. Moreover, PAD4 ${ }^{\text {LLD }}$ is not sufficient to interact with EDS1, indicating the PAD4-EP domain is required for stable heterodimerization. These data provide molecular evidence that PAD4 has domain-specific functions.
\end{abstract}

Keywords: aphid, basal immunity, EDS1, ETI, PAD4

To colonize plants, pathogenic microbes and pests (such as aphids or nematodes) deliver susceptibility factors called effectors to the host, which target defenses and reprogram cells to promote infection or infestation. Many host-adapted biotrophic and hemibiotrophic pathogens deploy effectors to disable pathogen- or microbe-associated molecular pattern-triggered immunity (PTI) mediated by cell surface-resident receptors (Boutrot and Zipfel 2017; Dodds and Rathjen 2010; Jones and

Current address for Deepak D. Bhandari: Plant Research Laboratory, Michigan State University, East Lansing, MI, U.S.A.

${ }^{\dagger}$ Corresponding author: J. E. Parker; parker@mpipz.mpg.de

Funding: This work was supported by the Max Planck Society and Deutsche Forschungsgemeinschaft grant CRC670 and DFG/Agence Nationale de la Recherche Trilateral 'RADAR' grant ANR-15-CE20-0016-01, and through a scholarship from the University of North Texas to M. Patel.

*The $\boldsymbol{e}$-Xtra logo stands for "electronic extra" and indicates that four supplementary figures and one supplementary table are published online.

The author(s) declare no conflict of interest.

() 2020 The American Phytopathological Society
Dangl 2006). These microbes encounter two further important immunity barriers. One is conferred by intracellular nucleotidebinding leucine-rich repeat (NLR) receptors recognizing interference by specific effectors (Jones et al. 2016). NLR activation leads to effector-triggered immunity (ETI) involving the rapid transcriptional mobilization of resistance pathways and, often, localized host cell death, which limits pathogen infection (Bhandari et al. 2019; Cui et al. 2015; Mine et al. 2017). NLR-mediated immune responses are also effective against probing insects and nematodes (Milligan et al. 1998; Rossi et al. 1998; Villada et al. 2009; Wroblewski et al. 2007). A second barrier, called basal immunity, slows virulent pathogen growth and disease progression by eliciting a weak immune response (Cui et al. 2015, 2017; Jones and Dangl 2006). Although the precise activation mechanism for postinfection basal immunity is not known, in Arabidopsis, it requires several ETI signaling components (Century et al. 1995; Feys et al. 2001; Glazebrook et al. 1997; Parker et al. 1996) and is proposed to be the culmination of weak NLR-triggered ETI combined with residual PTI (Cui et al. 2017; Gantner et al. 2019).

In Arabidopsis, the nucleocytoplasmic immune regulator PAD4 (PHYTOALEXIN DEFICIENT4) signals in both ETI and basal immunity by stimulating production of the defense hormone salicylic acid (SA) and antimicrobial molecules, which limit pathogen growth (Glazebrook et al. 1997; Jirage et al. 1999; Wiermer et al. 2005; Zhou et al. 1998). PAD4 is a member of a small family (the EDS1 family) of sequence-related immunity regulators, comprising also EDS1 (ENHANCED DISEASE SUSCEPTIBILITY1) and SAG101 (SENESCENCE-ASSOCIATED GENE101) (Feys et al. 2005; Lapin et al. 2019). Arabidopsis EDS1 and PAD4 function together in conferring ETI governed by a subclass of NLRs with N-terminal Toll-interleukin 1 receptor (TIR) domains (known as TIR-NLRs or TNLs) (Feys et al. 2005; Jones et al. 2016; Wagner et al. 2013). Genetic and molecular studies in Arabidopsis revealed that activated TNL receptors stimulate EDS1PAD4 basal immunity activity to transcriptionally boost SA and other defense responses and repress antagonistic jasmonic acid (JA) hormone pathways (Bhandari et al. 2019; Cui et al. 2017, 2018). In Arabidopsis, the EDS1-PAD4 transcriptional reprogramming function in pathogen immunity requires a nuclear EDS1 pool (Bartsch et al. 2006; Cui et al. 2017; García et al. 2010; Stuttmann et al. 2016).

EDS1, PAD4, and SAG101 each possess an N-terminal lipase-like domain (LLD) with an $\alpha / \beta$ hydrolase topology resembling eukaryotic class-3 lipase enzymes (Rauwerdink and 
Kazlauskas 2015; Wagner et al. 2013; Wang et al. 2018) and a structurally unique C-terminal EP (EDS1-PAD4) domain consisting of $\alpha$-helical bundles (PFAM database PF18117) (Wagner et al. 2013). The EDS1 and PAD4 but not SAG101 LLDs have a canonical Ser-Asp-His (S-D-H) catalytic triad that is characteristic for $\alpha / \beta$ hydrolases (Wagner et al. 2013). The serine is part of a lipase GXSXG motif, which is conserved in EDS1 and PAD4 proteins across seed plant (angiosperm and gymnosperm) species (Lapin et al. 2019; Wagner et al. 2013). Strikingly, the S-D-H residues were found to be dispensable for EDS1 and PAD4 signaling in Arabidopsis TNL-mediated ETI and basal immunity, indicative of a noncatalytic mechanism in pathogen resistance. These and structural analyses support the hypothesis that the Arabidopsis EDS1 family proteins are pseudoenzymes (Louis et al. 2012a; Voss et al. 2019; Wagner et al. 2013).

EDS1 forms stable and mutually exclusive heterodimers with PAD4 or SAG101, consistent with distinct roles of these two EDS1 complexes in immunity (Lapin et al. 2019; Rietz et al. 2011; Wagner et al. 2013). Based on a structural model of the EDS1-PAD4 heterodimer generated from the AtEDS1AtSAG101 crystal structure, analysis showed that the juxtaposed LLDs are major drivers of heterodimerization, likely promoting association of the aligned EP domains to form a cavity (Wagner et al. 2013). The AtEDS1 ${ }^{\text {LLD }}$ alone, although stable, did not confer pathogen resistance, indicating that its EP domain is crucial for immune signaling activity (Wagner et al. 2013). Further structure-based analysis identified an AtEDS1 EP-domain surface lining the EDS1-PAD4 heterodimer cavity that is essential for the rapid transcriptional reprogramming of host cells in Arabidopsis TNL ETI (Bhandari et al. 2019; Lapin et al. 2019).

In Arabidopsis, PAD4 mediates resistance to green peach aphid (GPA, Myzus persicae Sülzer) independently of EDS1 and SAG101 (Pegadaraju et al. 2005, 2007). GPA population growth was higher on Arabidopsis pad4 compared with wild type (WT) and eds1, sag101, or eds1/sag101 mutant plants (Pegadaraju et al. 2007). GPA population growth on pad4 was similar to mutants of other components in GPA resistance, ACTIN-DEPOLYMERIZING FACTOR 3 (ADF3) and TREHALOSE PHOSPHATE SYNTHASE11 (TPS11) (Mondal et al. 2018; Singh et al. 2011). Notably, PAD4-mediated defenses against GPA were found to not involve SA or camalexin production (Pegadaraju et al. 2005). Moreover, in contrast to basal immunity and ETI, resistance to GPA was dependent on the S$\mathrm{D}-\mathrm{H}$ predicted catalytic triad residues PAD $4^{\mathrm{S} 118}$ and PAD4 ${ }^{\mathrm{D} 178}$ but not PAD4 ${ }^{\mathrm{H} 229}$ (Louis et al. 2012a; Wagner et al. 2013). These different requirements suggest that PAD4 functions in immunity as a heterodimer with EDS1 are distinct from its function in resistance to GPA.

To gain a deeper insight into the molecular function of PAD4, we investigate, here, the properties of the N-terminal PAD4 LLD (PAD4 ${ }^{\mathrm{LLD}}$ ) in resistance to GPA and pathogen immunity. We show that the PAD4 ${ }^{\mathrm{LLD}}$ alone is sufficient to control GPA infestation independently of EDS1 association. By contrast, we find that the Arabidopsis PAD4 ${ }^{\mathrm{LLD}}$ is insufficient for EDS1dependent basal immunity and ETI, indicating that, like EDS1, the PAD4 EP domain is crucial for inducing immunity pathways. These results suggest that PAD4 can operate as a bipartite protein with the LLD and EP domains carrying out distinctive and separable roles in plant defense.

\section{RESULTS}

The PAD4 ${ }^{L L D}$ protein accumulates in planta but does not interact with EDS1.

AtEDS1-AtPAD4 heterodimer formation is driven chiefly by an N-terminal EDS1 hydrophobic loop $\left(\alpha\right.$-helix H) $\left(\mathrm{EDS}^{\mathrm{LLIF}}\right)$ and the juxtaposed PAD4 ${ }^{\mathrm{MLF}}$ motif (Fig. 1A, B, and C) (Feys et al. 2001; Wagner et al. 2013). To test PAD $4^{\text {LLD }}$ properties, we generated an AtPAD4 ${ }^{\mathrm{LLD}}$ protein (residues 1 to 299) (Fig. 1A, blue). Transient overexpression of green fluorescent protein (GFP)-tagged PAD4 ${ }^{\mathrm{LLD}}$ in Nicotiana benthamiana produced a stable protein that did not coimmunoprecipitate FLAG-tagged EDS1, whereas full-length GFP-PAD4 did (Fig. 1D). Similarly, PAD4 ${ }^{\text {LLD }}$ failed to interact with EDS1 in a $N$. benthamiana split luciferase assay (Supplementary Fig. S1). These data suggest that a stable interaction between PAD4 and EDS1 in planta requires part or all of the PAD4 EP domain in addition to the LLD interface.

To investigate PAD4 ${ }^{\mathrm{LLD}}$ properties in Arabidopsis, we introduced WT PAD4 (pPAD4:strepII-YFP-cPAD4 $4^{W T}$ ) or PAD4 $4^{L L D}$ (pPAD4:strepII-YFP-cPAD4 ${ }^{L L D}$ ) constructs into a pad4-1/sag1013 mutant (Col-0 accession). PAD4 ${ }^{\mathrm{LLD}}$ in two independent stable transgenic lines exhibited a nucleocytoplasmic localization similar to $\mathrm{PAD}^{\mathrm{WT}}$ at $24 \mathrm{~h}$ postinfection (hpi) with Pseudomonas syringae pv. tomato DC3000 expressing the effector avrRps4 (hereafter called avrRps4) (Supplementary Fig. S2). Delivery of avrRps4 by $P$. syringae pv. tomato triggers ETI in Col-0 mediated by the receptor pair RRS1-S/RPS4 (RESISTANCE TO RALSTONIA SOLANACEARUM1-S/RESISTANCE TO PSEUDOMONAS SYRINGAE4) (Birker et al. 2009; Heidrich et al. 2011; Narusaka et al. 2009; Saucet et al. 2015). The PAD4 ${ }^{\text {LLD }}$ distribution is in line with previously described nucleocytoplasmic localizations of EDS $1^{\mathrm{LLD}}$ and PAD4 ${ }^{\mathrm{LLD}} / \mathrm{SAG} 101^{\mathrm{EP}}$ domain chimeras in planta (Lapin et al. 2019; Wagner et al. 2013). PAD4 ${ }^{\text {LLD }}$ protein was also immuno-detected in leaf samples treated with avrRps4, although at much lower levels compared with PAD4 ${ }^{\mathrm{WT}}$ lines (Fig. 1E). This contrasts with similar PAD4 ${ }^{\mathrm{LLD}}$ and PAD4 ${ }^{\mathrm{WT}}$ accumulation in $N$. benthamiana transient assays (Fig. 1D). Lower PAD4 ${ }^{\text {LLD }}$ protein accumulation than PAD4 ${ }^{\text {WT }}$ in mockand avrRps4-treated Arabidopsis leaves can be attributed, in part, to lower accumulation of PAD4 transcripts in the PAD $4{ }^{\mathrm{LLD}}$ compared with PAD4 ${ }^{\mathrm{WT}}$ transgenic lines (Fig. 1F). Hence, the LLD domain of PAD4 is sufficient to maintain a WT-like nucleocytoplasmic localization, but loss of the EP domain substantially reduces PAD4-EDS1 interaction and PAD4 steady-state levels in Arabidopsis.

\section{Expression of PAD4 ${ }^{\text {LLD }}$ confers GPA resistance.}

PAD4 acts independently of EDS1 to restrict aphid infestation, and this function is dependent on the PAD4 ${ }^{\text {LLD }}-$ lo- $^{-}$ cated $\mathrm{S}^{118}$ and $\mathrm{D}^{178}$ predicted $\alpha / \beta$-hydrolase catalytic triad residues (Fig. 1A and B) (Louis et al. 2012a; Pegadaraju et al. 2007). Since PAD4 ${ }^{\mathrm{LLD}}$ accumulates in Arabidopsis, we tested whether the PAD4 ${ }^{\mathrm{LLD}}$ alone is able to resist GPA infestation. Consistent with earlier data (Louis et al. 2012a; Pegadaraju et al. 2007), pad4-1, pad4-1/sag101-3, and a PAD4 ${ }^{\text {S118A }}$ line (in pad4-1/eds1-2/EDS1 $^{\text {SDH }}$ ) (Wagner et al. 2013) permitted a significant increase in aphid population size compared with Col-0 in a no-choice bioassay, indicating compromised resistance to GPA infestation (Fig. 2). The PAD4 ${ }^{\mathrm{LLD}}$ lines resisted GPA to similar levels as PAD4 ${ }^{\mathrm{WT}}$ and Col-0, even though they expressed very low PAD4 ${ }^{\text {LLD }}$ amounts (Fig. 2). Hence, low steady-state accumulation of PAD4 ${ }^{\mathrm{LLD}}$ protein (Fig. 1E) is sufficient to counter GPA infestation in Arabidopsis, implying that PAD $4^{\text {LLD }}$ has an inplanta activity. Based on these data, we conclude that PAD4 ${ }^{\mathrm{LLD}}$ is a stable protein entity able to confer GPA resistance.

\section{Arabidopsis ETI and basal pathogen immunity require full-length PAD4.}

Since PAD $4^{\mathrm{LLD}}$ transgenic plants were as resistant as Col0 against GPA, we tested if the PAD $4{ }^{\text {LLD }}$ domain also functions in one or both basal and TNL-triggered pathogen immunity. For this, we measured TNL ETI, using the biotrophic pathogen 

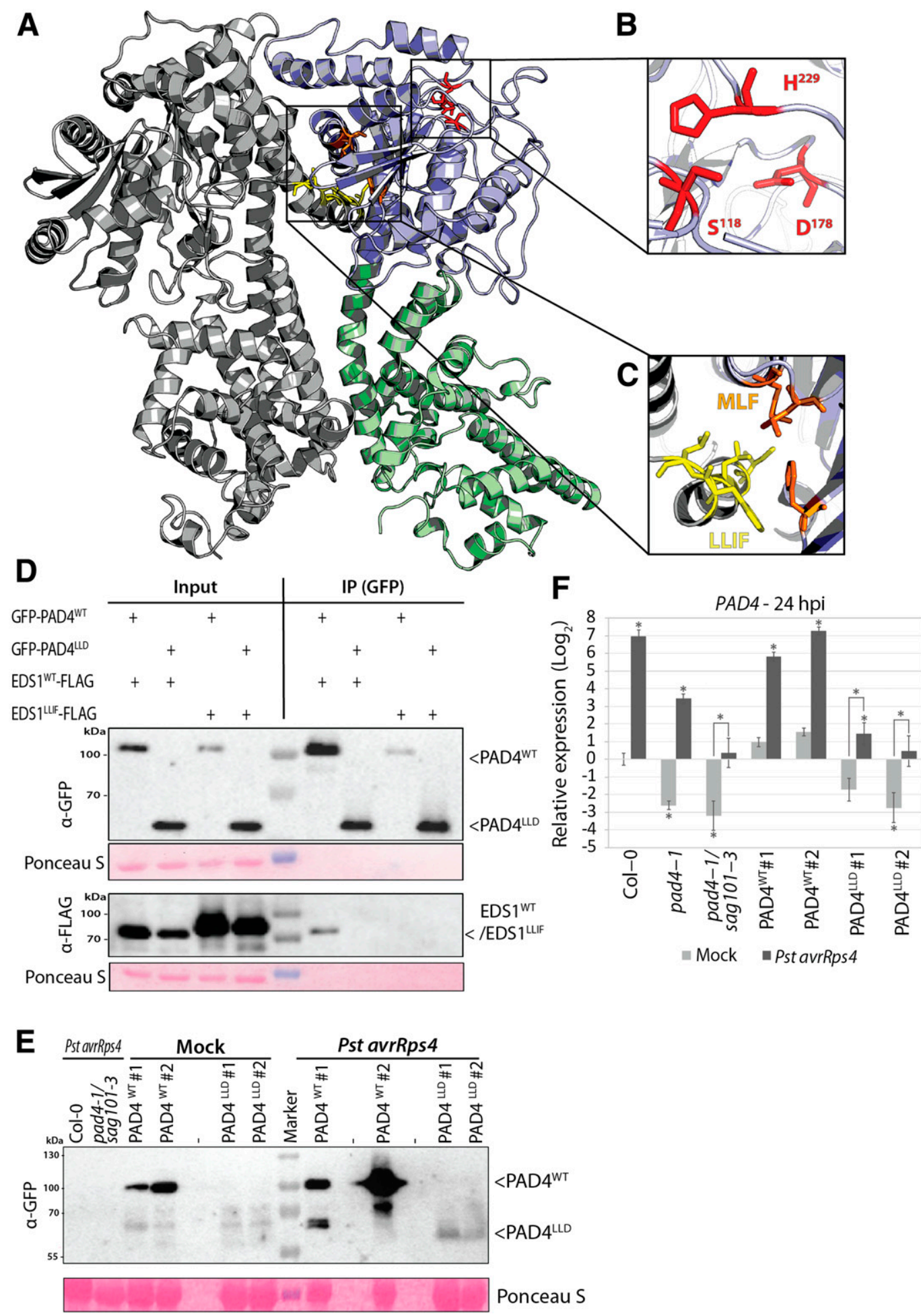

Fig. 1. The PAD4 lipase-like domain $\left(\mathrm{PAD} 4{ }^{\mathrm{LLD}}\right)$ accumulates in planta but does not interact with EDS1. A, A EDS1-PAD4 heterodimer model, based on the AtEDS1-AtSAG101 crystal structure (Wagner et al. 2013). EDS1 (gray), PAD4 (LLD) (blue) and PAD4 C-terminal EDS1-PAD4 domain (green) are represented in cartoon format. B, PAD4 catalytic triad residues S118, D178, and H229 in the LLD are shown as red sticks. C, EDS1-PAD4-interacting motifs $\mathrm{EDS} 1^{\mathrm{LLIF}}$ and PAD4 ${ }^{\mathrm{MLF}}$ are colored with yellow and orange sticks, respectively. D, Coimmunoprecipitation (green fluorescent protein [GFP]-trap) of GFP$\mathrm{PAD} 4{ }^{\mathrm{WT}} / \mathrm{PAD} 4{ }^{\mathrm{LLD}}$ with EDS $1^{\mathrm{WT}} / \mathrm{EDS} 1^{\mathrm{LLIF}}-3 \mathrm{xFLAG}$ transiently coexpressed in Nicotiana benthamiana leaves (using $35 \mathrm{~S}: \mathrm{GFP}-\mathrm{PAD} 4^{\mathrm{WT}} / \mathrm{PAD}{ }^{\mathrm{LLD}}$ and $35 \mathrm{~S}$ : $\mathrm{EDS} 1^{\mathrm{WT}} / \mathrm{EDS} 1^{\mathrm{LLIF}}-3 \mathrm{xFLAG}$ constructs, respectively). On the left, all proteins are expressed in the input; on the right, in the immunoprecipitation (IP) fraction, only EDS $1^{\mathrm{WT}}$ coimmunoprecipitates with PAD4 $4^{\mathrm{WT}}$ (positive control). The noninteracting variant EDS1 ${ }^{\mathrm{LLIF}}$ does not coimmunoprecipitate with PAD4 ${ }^{\mathrm{WT}}$ (negative control) (Wagner et al. 2013). PAD4 ${ }^{\mathrm{LLD}}$ does not coimmunoprecipitate EDS1 ${ }^{\mathrm{WT}}$ or EDS1 $1^{\mathrm{LLIF}}$. A representative image from three independent experiments is shown. E, PAD4 accumulation in independent stable transgenic Arabidopsis lines expressing yellow fluorescent proteins YFP-PAD4 ${ }^{\mathrm{WT}}$ and

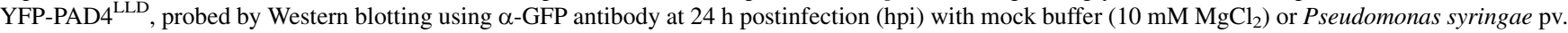
tomato avrRps4 treatments. A representative image from three independent experiments is shown. F, PAD4 transcript abundance was determined by reverse transcription-quantitative PCR at 24 hpi in mock buffer- or P. syringae pv. tomato avrRps4-treated samples of the indicated Arabidopsis lines. Data are pooled from three independent experiments, each with two to three biological replicates $(n=6$ to 9$)$. Bars represent means of three experimental replicates \pm standard error. Relative expression and significance level is set to Col-0 mock-treated samples. Asterisk indicates $P<0.01$, one-way analysis of variance with multiple testing correction using Tukey honestly significant difference. 
Hyaloperonospora arabidopsidis isolate EMWA1, which is recognized in Col-0 by the TNL RPP4 (RESISTANCE TO PERONOSPORA PARASITICA4) (Asai et al. 2018; van der Biezen et al. 2002). Col-0, PAD4 ${ }^{\mathrm{WT}}$ and PAD4 ${ }^{\text {S118A }}$ lines were fully resistant to $H$. arabidopsidis EMWA1, as measured by conidiophore production (Fig. 3A). By contrast, PAD4 ${ }^{\text {LLD }}$ transgenic lines were fully susceptible with conidiophore production and macroscopic disease and microscopic $H$. arabidopsidis colonization phenotypes resembling a pad41/sag101-3 mutant (Fig. 3A; Supplementary Fig. S3).

Further, we tested PAD4 ${ }^{\mathrm{LLD}}$ function in TNL (RRS1S/RPS4) ETI to avrRps4 and in basal immunity to virulent P. syringae pv. tomato DC3000. In basal immunity, pad4-1 is as susceptible as pad4-1/sag 101-3, while in ETI, pad4-1 displays intermediate susceptibility between Col-0 and pad4-1/sag1013 (Fig. 3B and C) (Feys et al. 2005; Wagner et al. 2013). In line with published data, PAD4 ${ }^{\mathrm{S} 118 \mathrm{~A}}$ was as resistant as Col-0 and PAD $4^{\text {WT }}$ in both basal immunity and ETI (Fig. 3B and C), consistent with previous findings that the PAD4 S-D-H predicted catalytic triad is not required for pathogen immunity (Louis et al. 2012a; Wagner et al. 2013). PAD4 ${ }^{\text {LLD }}$ lines were fully susceptible to $P$. syringae pv. tomato DC3000 and avrRps4, with bacterial titers comparable to pad4-1/sag 101-3 (Fig. 3B and C), indicating that PAD4 ${ }^{\mathrm{LLD}}$ is not able to confer basal immunity or ETI. Also, PAD4 ${ }^{\text {LLD }}$-expressing plants and pad4-1/sag101-3 failed to induce expression of defense marker genes 24 hpi with avrRps4, indicating that PAD $4^{\mathrm{LLD}}$ is unable to signal in TNL ETI (Fig. 3D, E, and F; Supplementary Fig. S4). Taken together, the $H$. arabidopsidis and $P$. syringae pv. tomato infection data show that PAD $4^{\mathrm{LLD}}$ is nonfunctional in pathogen basal immunity and ETI, in stark contrast to its resistance activity against GPA.

\section{DISCUSSION}

PAD4 controls Arabidopsis defenses against pathogens and aphids, playing major roles with EDS1 in basal immunity and ETI and an EDS1-independent role in resistance to GPA (Bhandari et al. 2019; Cui et al. 2017, 2018; Glazebrook et al. 1997; Lapin et al. 2019; Louis et al. 2012a; Pegadaraju et al. 2007; Rietz et al. 2011; Wagner et al. 2013). In this study, we investigated the contribution of the PAD4 $4^{\mathrm{LLD}}$ to these different defense outputs. Analysis of PAD4 ${ }^{\text {LLD }}$ in planta shows that it accumulates to much lower levels than full-length PAD4 and has lost binding to EDS1. In Arabidopsis, PAD4 ${ }^{\mathrm{LLD}}$ confers complete GPA resistance (Fig. 2) but is nonfunctional in resistance to $H$. arabidopsidis and $P$. syringae pv. tomato pathogens (Fig. 3). Thus, PAD4 appears to rely solely on its LLD for controlling GPA infestation, whereas its LLD and EP domains are necessary for ETI and basal immunity against bacterial and oomycete pathogens. These data suggest there are domain-specific signaling functions of Arabidopsis PAD4.

Recent studies suggest that the N-terminal LLDs of Arabidopsis EDS1 and PAD4 act as a scaffold, enabling the Cterminal EP domains to interact and orchestrate downstream immune signaling as a heterodimer (Bhandari et al. 2019; Lapin et al. 2019; Wagner et al. 2013). By testing the PAD4 ${ }^{\text {LDD }}$ without its EP domain, our data show that PAD4, like EDS1 (Bhandari et al. 2019), requires its EP domain for immunity signaling. By contrast, the PAD $4^{\mathrm{LLD}}$ is sufficient to limit GPA proliferation, thus highlighting a role of the PAD4 ${ }^{\text {LLD }}$ and its $\alpha / \beta$-hydrolase catalytic triad as a minimal functional unit in GPA resistance. AtEDS1 and AtPAD4 proteins mutually stabilize each other (Feys et al. 2001, 2005; Rietz et al. 2011; Wagner et al. 2013). The fact that interaction between PAD4 ${ }^{\text {LLD }}$ and EDS1 is greatly diminished, compared with interaction between full-length PAD4 and EDS1 (Fig. 1D), tallies with the observation that PAD4-dependent GPA resistance is independent of EDS1 (Pegadaraju et al. 2007). It is unclear why low PAD4 ${ }^{\mathrm{LLD}}$ accumulation is sufficient for aphid resistance. It is possible that because PAD $4{ }^{\mathrm{LLD}}$ does not interact with EDS1, more PAD4 ${ }^{\mathrm{LLD}}$ protein is available for the LLD activity in aphid resistance, as it is not held in a pool with EDS1, although absence of EDS1 did not alter GPA resistance (Pegadaraju et al. 2007). Another possibility is that PAD4 ${ }^{\text {LLD }}$ accumulates at aphid feeding sites, since PAD4 expression was shown to be induced here (Louis et al. 2012b). The site of action of PAD4 ${ }^{\mathrm{LLD}}$ in tissues and cells is worth examining in future studies.

The PAD $4^{\text {LLD }}$ adopts an $\alpha / \beta$ hydrolase fold with a core $S-D-$ $\mathrm{H}$ predicted catalytic triad. The $\alpha / \beta$ hydrolase family catalyzes a variety of enzymatic reactions such as esterification, hydrolysis and acyl transfer (Rauwerdink and Kazlauskas 2015). The S-D-H predicted catalytic triad of PAD4 is dispensable for immune signaling against $H$. arabidopsidis and $P$. syringae pv. tomato but required for GPA resistance (Louis et al. 2012a; Wagner et al. 2013). In the AtPAD4 structural model, this triad of residues is solvent-accessible (Fig. 1A and B), suggesting a plausible catalytic function. However, this applies only to Brassicaceae PAD4 proteins, as, beyond the Brassicaceae clade, PAD4 contains an insertion that forms a "lid" covering the S-D-H triad, similar to that in AtEDS1, rendering it inaccessible to the solvent (Wagner et al. 2013). Such helical loop structures extending from the $\beta$-sheet scaffold have been found to regulate the enzymatic activity of inactive-state triacylglycerol lipases (Khan et al. 2017). Hence, it is possible that the PAD4 S-D-H triad functions differently outside the

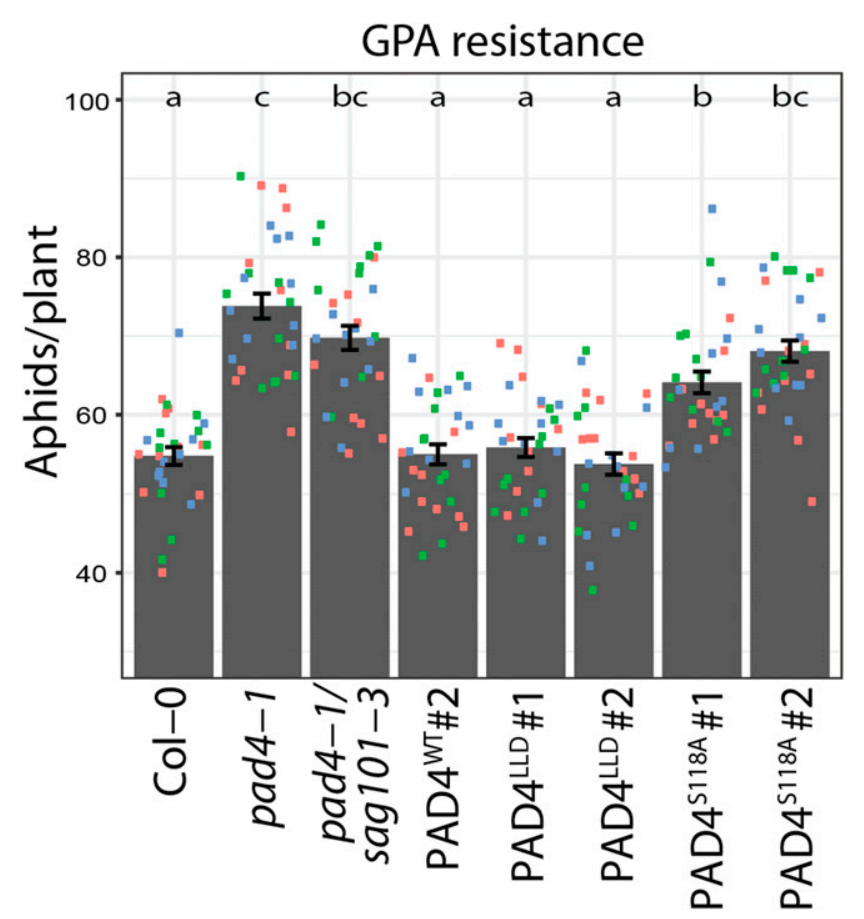

Fig. 2. The PAD4 lipase-like domain $\left(\mathrm{PAD} 4^{\mathrm{LLD}}\right)$ is sufficient for green peach aphid (GPA) resistance. Numbers of GPA per plant at 11 days postinfestation in a no-choice assay. Data are pooled from three independent experiments, each with ten biological replicates per experiment $(n=30)$. Squares of the same color represent ten biological replicates in an independent experiment. Bars represent mean of three experimental replicates \pm standard error. Differences between genotypes were determined using analysis of variance (Tukey honestly significant difference, $P<0.01$ ), letters indicate significance class. 
A

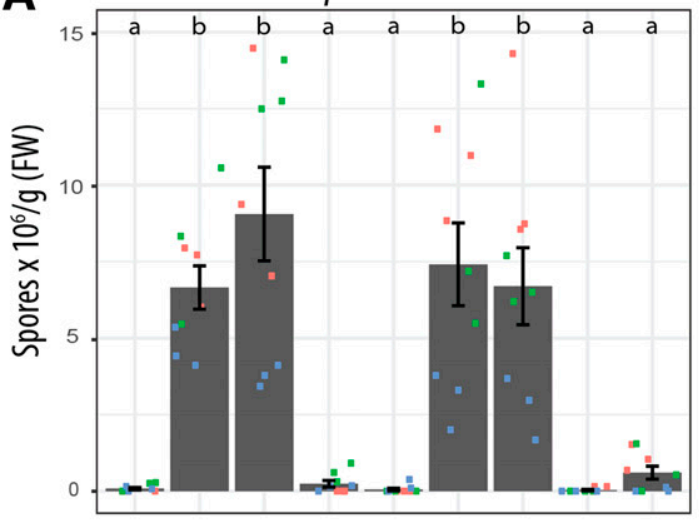

B

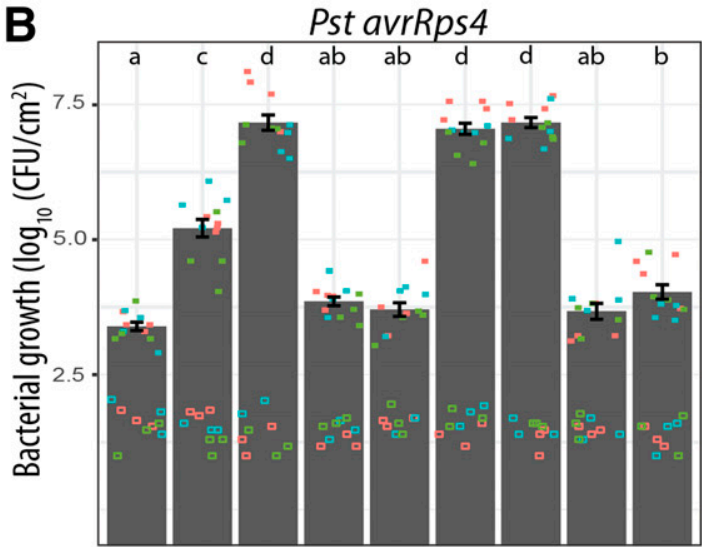

C

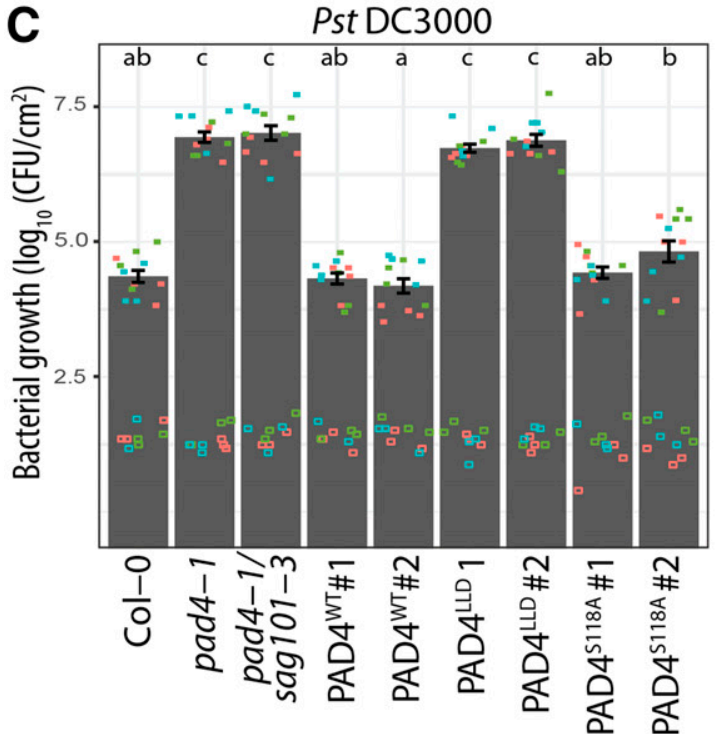

D

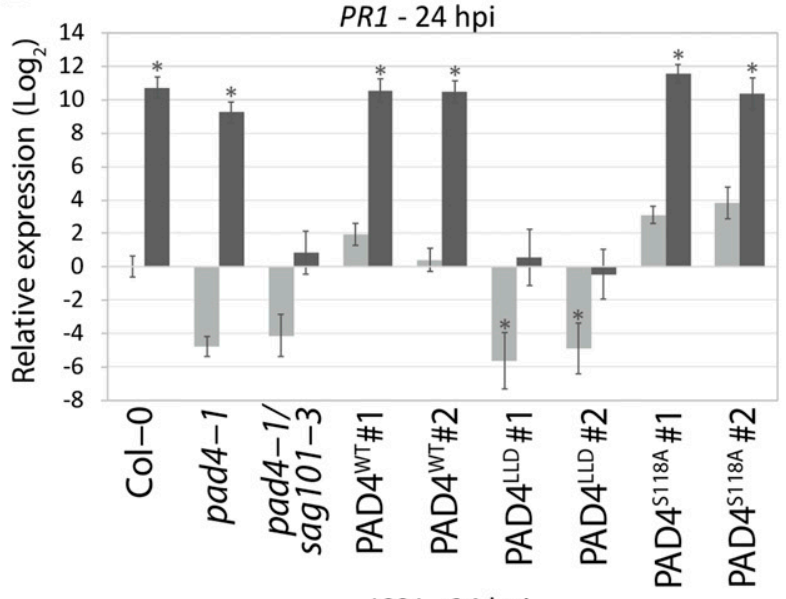

ICS1 - $24 \mathrm{hpi}$
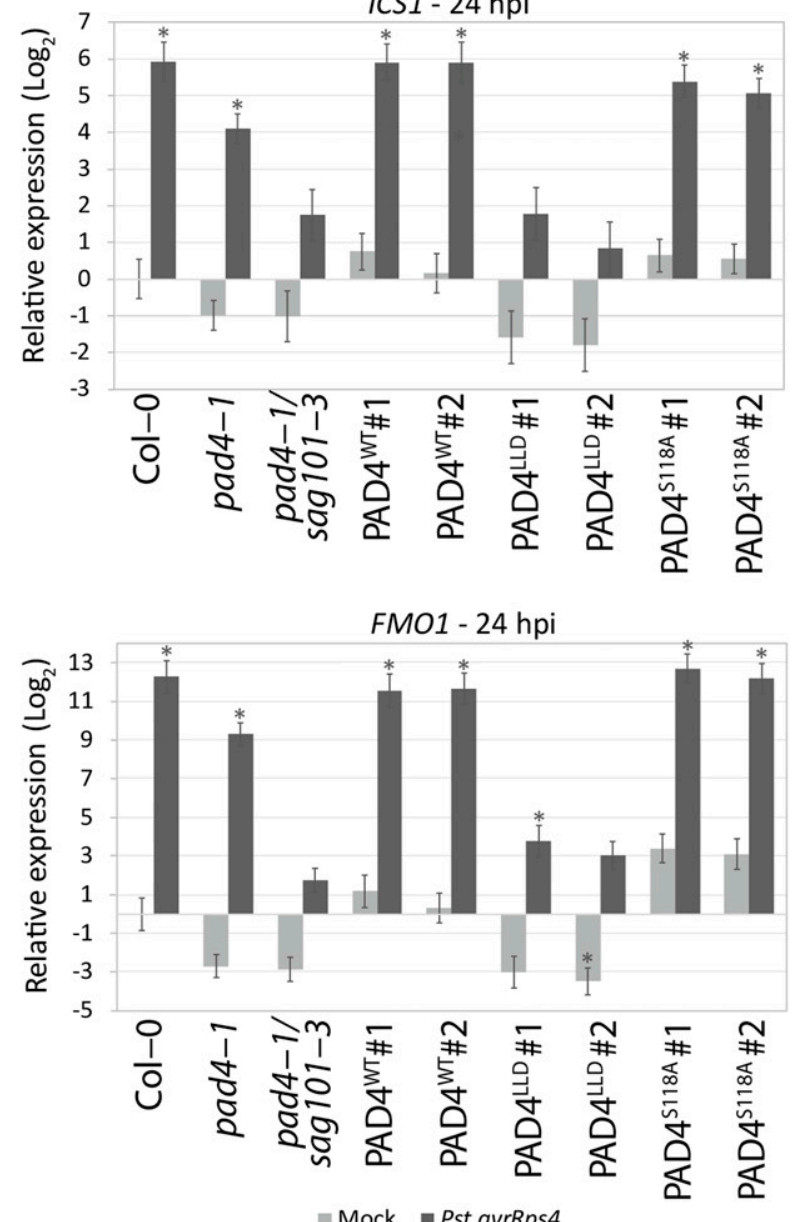

Fig. 3. The PAD4 lipase-like domain $\left(\mathrm{PAD} 4^{\mathrm{LLD}}\right.$ ) is not functional in Arabidopsis effector-triggered immunity (ETI) and basal immunity. A, A toll interleukin 1 receptor nucleotide-binding leucine-rich repeat (TNL) (RPP4) ETI assay in Arabidopsis independent transgenic lines with wild type and mutant controls, as indicated. Hyaloperonospora arabidopsidis ( $\mathrm{Hpa}$ ) EMWA1 conidiospores on leaves were quantified at 6 days postinfection (dpi) in three independent experiments (squares; $n=9$ ). Col-0 (resistant), pad4-1 (susceptible), and pad4-1/sag101-3 (susceptible) functioned as controls. Squares of the same color represent three biological replicates in an independent experiment. Bars represent mean of three experimental replicates \pm standard error (SE). Differences between genotypes were determined using analysis of variance (ANOVA) (Tukey honestly significant difference [HSD], $P<0.01$ ), letters indicate significance class. B, TNL (RRS1-S/RPS4) ETI assay in the same Arabidopsis independent transgenic and control lines as in A. Four-week-old Arabidopsis plants were syringe-infiltrated with Pseudomonas syringae pv. tomato $(P s t)$ avrRps4 (optical density at $600 \mathrm{~nm}\left[\mathrm{OD}_{600}\right]=0.0005$ ) and bacterial titers were determined at 0 dpi (empty squares; $n=8$ to 9) and 3 dpi (filled squares; $n=11$ to 12). Squares of the same color represent 2 to 3 (day 0 ) or 3 to 4 (day 3 ) biological replicates in an independent experiment. Bars represent mean of three experimental replicates \pm SE. Differences between genotypes were determined using ANOVA (Tukey HSD, $P<0.01)$, letters indicate significance class. C, Infection assay was performed with basal immunity triggering $\mathrm{DC}_{3000}\left(\mathrm{OD}_{600}=0.0005\right)$. Experimental setup and statistical analysis as described in B. D, Transcript abundance determined by reverse transcription-quantitative PCR in 4-week-old Arabidopsis plants syringe-infiltrated with either buffer (mock, gray bars) or avrRps4 (black bars) ( $24 \mathrm{~h}$ postinfection). Data are pooled from three independent experiments, with two to three biological replicates per experiment ( $n=6$ to 9). PATHOGENESIS RELATED1 (PR1), ISOCHORISMATE SYNTHASE1 (ICS1), and FLAVIN MONOOXYGENASE1 (FMO1) transcript abundances were measured relative to ACTIN2 (ACT2). Relative expression and significance level is set to Col-0 mock-treated samples. Differences between genotypes were determined using ANOVA (Tukey HSD), asterisks indicate $P<0.01$. 
Brassicaceae clade (Wagner et al. 2013). Critically, all three residues in the catalytic triad are required for hydrolase activity (Rauwerdink and Kazlauskas 2015). Since loss of $\mathrm{H}^{229}$ does not affect AtPAD4-mediated deterrence of GPA (Louis et al. 2012a), it is unlikely that PAD4 involvement in Arabidopsis defense against the GPA requires a canonical hydrolase activity.

Alternatively, the S-D-H triad in PAD4 could function as a receptor ligand-binding domain, a common feature of $\alpha / \beta$ hydrolase fold proteins (Mindrebo et al. 2016). For example, the Arabidopsis karrikin receptor AtKAI2 (KARRIKIN INSENSITIVE 2) uses its catalytically inactive S-D-H triad for ligand recognition (Guo et al. 2013). Catalytically inactive rice (Oryza sativa) and AtGID1 (GIBBERELLIN [GA]-INSENSITIVE DWARF1) use a modified triad (S-D-V) to bind bioactive GA molecules, indicating that the histidine, which is required for catalytic activity, can be replaced by another residue for functional ligand binding (Murase et al. 2008; Rauwerdink and Kazlauskas 2015; Shimada et al. 2008). Upon binding to GA, a conformational change in AtGID1 results in the assembly of a $\mathrm{SCF}^{\mathrm{GID} 1}$ (SKP-Cullin-F-box ${ }^{\mathrm{GID} 1}$ ) complex and ubiquitination of DELLA proteins marking them for proteasome-mediated degradation (Murase et al. 2008). Together with the data presented here, these examples highlight the possibility that the PAD $4^{\mathrm{LLD}}$ domain serves as a ligand-binding surface in a protein signaling complex rather than a lipase. A recent study of the AtEDS1 monomer structure reinforces the view that Arabidopsis EDS1 is a pseudoenzyme (Voss et al. 2019). Further insights to the mechanism by which PAD4 mediates GPA resistance will help to determine whether PAD $4{ }^{\mathrm{LLD}}$ functions as a lipase or a receptor in GPA resistance.

The inactivity of PAD4 ${ }^{\mathrm{LLD}}$ in basal and ETI is unlikely to be attributed to PAD4 ${ }^{\text {LLD }}$ instability, as it is sufficient for resistance against GPA, unless the PAD $4^{\text {LLD }}$ fails to reach sufficient amounts needed for pathogen resistance in certain cells or tissues. Very low levels of protein were sufficient for EDS1 function in pathogen immunity (Bhandari et al. 2019; Stuttmann et al. 2016; Wagner et al. 2013), and we presume this is also the case for PAD4, since EDS1 and PAD4 are functional as a heterodimer. A more plausible explanation for the susceptibility of PAD4 ${ }^{\text {LLD }}$ might be i) its inability to form a heterodimer with EDS1 (Fig. 1A and D) (Wagner et al. 2013) and ii) the lack of an EP domain. Both are essential in EDS1 for immunity to $P$. syringae pv. tomato and $H$. arabidopsidis infection and for rapid transcriptional upregulation of defense genes in ETI against avrRps4 (Bhandari et al. 2019; Lapin et al. 2019; Wagner et al. 2013). The EDS1 EP domain interface lining a cavity formed with PAD4 in the heterodimer is necessary for Arabidopsis EDS1 signaling (Bhandari et al. 2019; Lapin et al. 2019). An aligned EP domain $\alpha$-helix was identified in the EDS1 heterodimer partner SAG101 as being essential for eliciting host cell death in TNL ETI responses (Gantner et al. 2019; Lapin et al. 2019). This also might be true for PAD4, because mutations at an EDS1-like surface in PAD4 lying outside the cavity did not compromise immunity (Bhandari et al. 2019). Future studies will test whether the PAD4 EP domain surface lining the heterodimer cavity is also crucial for EDS1-PAD4 pathogen immunity.

Our analysis of the LLD of Arabidopsis PAD4 demonstrates a domain-specific partitioning of defense functions, with the PAD $4^{\text {LLD }}$ being necessary and sufficient for limiting GPA infestation and the EP domain (with the LLD) mediating immunity signaling against $P$. syringae pv. tomato and $H$. arabidopsidis (Fig. 4). While the two PAD4 domains clearly have distinct roles, instability and inactivity of the PAD4 EP domain without the LLD makes it difficult to assess whether PAD4 is a bipartite immune regulator or moonlighting in GPA resistance. This study of the PAD $4^{\text {LLD }}$ paves the way for molecular dissection of the diverse roles of PAD4 in biotic stress resistance.

\section{MATERIALS AND METHODS}

Plant materials, growth conditions, and pathogen strains. Arabidopsis pad4-1, sag101-3, and eds1-2 mutants are in the Col-0 background and were previously described, as were pEDS1:EDS1 ${ }^{S D H}:: p P A D 4: P A D 4^{S 118 A}$ (in eds1-2/pad4-1) and pPAD4:StrepII-YFP-PAD4 (in pad4-1/sag101-3) transgenic lines (Bhandari et al. 2019; Wagner et al. 2013). Supplementary Table S1 lists primers used. $P$. syringae pv. tomato DC3000 and avrRps 4 were described previously (Cui et al. 2017). Plants were grown on soil in a controlled environment and insect-free chambers under a 10-h light and 14-h dark regime (photosynthetic active radiation, 100 to $150 \mu \mathrm{mol} / \mathrm{m}^{2} / \mathrm{s}$ ) at $22^{\circ} \mathrm{C}$ and $60 \%$ relative humidity.

\section{Pathogen infection assays.}

For bacterial growth assays, avrRps4 (optical density at $\left.600 \mathrm{~nm}\left[\mathrm{OD}_{600}\right]=0.0005\right)$ in $10 \mathrm{mM} \mathrm{MgCl}_{2}$ was handinfiltrated into leaves of 4-week-old plants. Bacterial titers were measured at $3 \mathrm{~h}$ postinfiltration (day 0 ) and 3 days, as described previously (Feys et al. 2005). Each biological replicate consisted of three leaf disks from different plants and data shown in each experiment are compiled from three to four biological replicates. Statistical analysis was performed using one-way analysis of variance with multiple testing correction using Tukey's honestly significant difference.

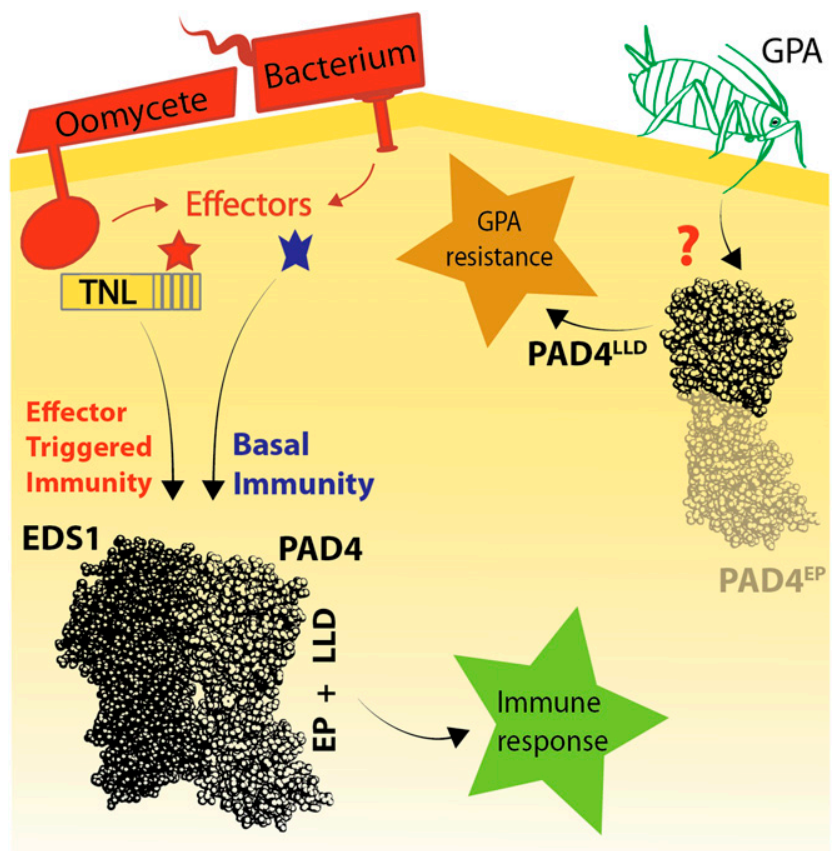

Fig. 4. Domain-specific roles of Arabidopsis PAD4 in immunity. Schematic showing separable activities of PAD4 lipase-like (PAD4 $\left.{ }^{\mathrm{LLD}}\right)$ and LLD + EP (C-terminal EDS1-PAD4) domains. Upon infection by bacteria or oomycetes, the EDS1-PAD4 heterodimer is activated via toll interleukin 1 receptor nucleotide-binding leucine-rich repeat domains (TNL) in effectortriggered immunity (ETI) or by other signals in basal immunity, leading to a pathogen immune response. PAD4 requires both the LLD and EP domains to function in basal immunity and ETI. In resistance to green peach aphids (GPA), PAD4 is activated through an unknown but EDS1-independent mechanism that restricts aphid infestation. PAD $4^{\text {LLD }}$ is sufficient to limit GPA independently of interaction with EDS1. 
For gene expression analysis, leaves of 4-week-old plants were hand-infiltrated with mock buffer $\left(10 \mathrm{mM} \mathrm{MgCl}_{2}\right)$ or bacteria $\left(\mathrm{OD}_{600}=0.005\right)$ and samples were taken at $24 \mathrm{hpi}$. $A C T 2$ was used as a reference gene (Supplementary Table S1). Data shown are results from three independent experiments each with two to three biological replicates.

For protein accumulation assays, leaves from 4-week-old plants were hand-infiltrated with buffer (mock, $10 \mathrm{mM} \mathrm{MgCl}$ ) or bacteria $\left(\mathrm{OD}_{600}=0.005\right)$ and samples were harvested by pooling leaves from at least three different plants.

$H$. arabidopsidis isolate EMWA1 was sprayed onto 2.5week-old plants at $4 \times 10^{4}$ spores per milliliter of distilled (d) $\mathrm{H}_{2} \mathrm{O}$. H. arabidopsidis infection structures and plant host cell death were visualized using lactophenol trypan blue staining (Muskett et al. 2002) and were imaged by light microscopy (Zeiss Axio Imager). To quantify $H$. arabidopsidis sporulation on leaves, three pots with approximately 10 plants per genotype were infected and were treated as a biological replicate. Plants were harvested at 6 days postinfection (dpi) and fresh weight was determined. Conidiospores were suspended in $5 \mathrm{ml}$ of $\mathrm{dH}_{2} \mathrm{O}$ and were counted under a light microscope, using a Neubauer counting chamber.

\section{Aphid no-choice bioassay.}

For each biological replicate five one-day-old nymphs were released onto the center of a 17-day-old plant. The total number of aphids (adult + nymphs) per biological replicate were counted 11 days postinfestation. Each independent experimental replicate consisted of 10 biological replicates per genotype (Nalam et al. 2018).

\section{Plasmid constructs.}

The pENTR/D-TOPO PAD4 vector used for site-directed mutagenesis was cloned from cDNA and is described (Wagner et al. 2013). PAD4 ${ }^{\mathrm{LLD}}$ was obtained by site-directed mutagenesis on $\mathrm{pENTR/D-TOPO} \mathrm{PAD4} \mathrm{according} \mathrm{to} \mathrm{the} \mathrm{QuikChangeII}$ site-directed mutagenesis manual (Agilent) (Supplementary Table S1). Mutated PAD4 and EDS1 entry clones (Bhandari et al. 2019; Wagner et al. 2013) were verified by sequencing and were recombined by an LR reaction into a pAM-PAT-based binary vector backbone (Witte et al. 2004). Split luciferase lines were created by LR reaction between Gateway-compatible split luciferase binary vectors (Gehl et al. 2011) and PAD4 and EDS1 entry clones (Bhandari et al. 2019; Wagner et al. 2013).

\section{Generation of transgenic Arabidopsis plants.}

Stable transgenic lines were generated by transforming a binary expression vector (containing Basta resistance) into Arabidopsis null mutant pad4-1/sag101-3 (Wagner et al. 2013), using Agrobacterium-mediated floral dipping (Agrobacterium tumefaciens GV3101 PMP90 RK) (Clough and Bent 1998). After selecting single-insert, homozygous transgenic lines, all lines were genotyped by sequencing for the presence of the correct PAD4 transgene (PAD4 ${ }^{\mathrm{WT}}, \mathrm{PAD} 4^{\mathrm{LLD}}$, or PAD4 ${ }^{\mathrm{S} 118 \mathrm{~A}}$ ) before performing pathogen assays.

\section{Transient expression in $N$. benthamiana.}

Transient expression in $N$. benthamiana was performed by coinfiltrating Agrobacterium cells carrying constructs at an $\mathrm{OD}_{600}$ of 0.4 TO 0.6 in a $1: 1$ ratio. Before syringe-infiltration, A. tumefaciens cells were incubated for $3 \mathrm{~h}$ at $28^{\circ} \mathrm{C}$ in induction buffer $(150 \mu \mathrm{M}$ acetosyringone, $10 \mathrm{mM}$ MES, pH5.6, $10 \mathrm{mM} \mathrm{MgCl} 2$ ) and were mixed at $650 \mathrm{rpm}$ in an Eppendorf thermomixer. $N$. benthamiana leaf samples were harvested at $3 \mathrm{dpi}$, were snap frozen in liquid nitrogen, and were stored at $-80^{\circ} \mathrm{C}$.

\section{Protein extraction, immunoprecipitation (IP), and Western blotting.}

Total leaf extracts were processed in extraction buffer (50 mM Tris, pH7.5, $150 \mathrm{mM} \mathrm{NaCl}, 10 \%$ [vol/vol] glycerol, $2 \mathrm{mM}$ EDTA, $5 \mathrm{mM}$ dithiothreitol, $0.1 \%$ Triton X-100, and protease inhibitor (Roche, 1 tablet per $50 \mathrm{ml}$ )). Lysates were centrifuged for $20 \mathrm{~min}, 21,000 \times g$ at $4^{\circ} \mathrm{C}$. Supernatant was used as input sample $(50 \mu \mathrm{l})$. IPs were conducted by incubating the input sample $(1.2 \mathrm{ml})$ with $10 \mu \mathrm{l}$ GFP TrapMA beads (Chromotek) for $3 \mathrm{~h}$ at $4^{\circ} \mathrm{C}$. Beads were collected using a magnetic rack and were washed four times in extraction buffer. Protein or IP samples were boiled at $96^{\circ} \mathrm{C}$ in $2 \times$ Laemmli buffer for $10 \mathrm{~min}$. Proteins were separated by sodium dodecyl sulfatepolyacrylamide gel electrophoresis and were analyzed by immunoblotting using $\alpha$-GFP (Sigma Aldrich) or $\alpha$-FLAG (Sigma Aldrich) primary antibodies and secondary antibodies coupled to horseradish peroxidase (Sigma Aldrich) for protein detection on blots.

\section{Luciferase assay.}

All tested coexpression constructs were transiently expressed on one leaf. Three leaf disks $(0.4 \mathrm{~cm}$ diameter $)$ from three independent leaves were pooled per biological replicate and were processed in reporter lysis buffer (Promega; $+150 \mathrm{mM}$ Tris, pH 7.5). Samples were mixed in a 1:1 ratio with substrate (Promega) and luminescence was measured. Absolute luminescence, i.e., absolute luciferase activity, was used as a proxy for protein-protein interaction intensity.

\section{ACKNOWLEDGMENTS}

We would like to thank K. Niefind for structural insights and N. Donnelly for help with editing the manuscript.

\section{LITERATURE CITED}

Asai, S., Furzer, O. J., Cevik, V., Kim, D. S., Ishaque, N., Goritschnig, S., Staskawicz, B. J., Shirasu, K., and Jones, J. D. G. 2018. A downy mildew effector evades recognition by polymorphism of expression and subcellular localization. Nat. Commun. 9:5192.

Bartsch, M., Gobbato, E., Bednarek, P., Debey, S., Schultze, J. L., Bautor, J., and Parker, J. E. 2006. Salicylic acid-independent ENHANCED DISEASE SUSCEPTIBILITY1 signaling in Arabidopsis immunity and cell death is regulated by the monooxygenase FMOI and the Nudix hydrolase NUDT7. Plant Cell 18:1038-1051.

Bhandari, D. D., Lapin, D., Kracher, B., von Born, P., Bautor, J., Niefind, K., and Parker, J. E. 2019. An EDS1 heterodimer signalling surface enforces timely reprogramming of immunity genes in Arabidopsis. Nat. Commun. 10:772.

Birker, D., Heidrich, K., Takahara, H., Narusaka, M., Deslandes, L., Narusaka, Y., Reymond, M., Parker, J. E., and O'Connell, R. 2009. A locus conferring resistance to Colletotrichum higginsianum is shared by four geographically distinct Arabidopsis accessions. Plant J. 60:602-613.

Boutrot, F., and Zipfel, C. 2017. Function, discovery, and exploitation of plant pattern recognition receptors for broad-spectrum disease resistance. Annu. Rev. Phytopathol. 55:257-286.

Century, K. S., Holub, E. B., and Staskawicz, B. J. 1995. NDR1, a locus of Arabidopsis thaliana that is required for disease resistance to both a bacterial and a fungal pathogen. Proc. Natl. Acad. Sci. U.S.A. 92: 6597-6601.

Clough, S. J., and Bent, A. F. 1998. Floral dip: A simplified method for Agrobacterium-mediated transformation of Arabidopsis thaliana. Plant J. 16:735-743.

Cui, H., Gobbato, E., Kracher, B., Qiu, J., Bautor, J., and Parker, J. E. 2017 A core function of EDS1 with PAD4 is to protect the salicylic acid defense sector in Arabidopsis immunity. New Phytol. 213:1802-1817.

Cui, H., Qiu, J., Zhou, Y., Bhandari, D. D., Zhao, C., Bautor, J., and Parker, J. E. 2018. Antagonism of transcription factor MYC2 by EDS1/PAD4 complexes bolsters salicylic acid defense in Arabidopsis effectortriggered immunity. Mol. Plant 11:1053-1066.

Cui, H., Tsuda, K., and Parker, J. E. 2015. Effector-triggered immunity: From pathogen perception to robust defense. Annu. Rev. Plant Biol. 66: 487-511. 
Dodds, P. N., and Rathjen, J. P. 2010. Plant immunity: Towards an integrated view of plant-pathogen interactions. Nat. Rev. Genet. 11: 539-548.

Feys, B. J., Moisan, L. J., Newman, M. A., and Parker, J. E. 2001. Direct interaction between the Arabidopsis disease resistance signaling proteins, EDS1 and PAD4. EMBO J. 20:5400-5411.

Feys, B. J., Wiermer, M., Bhat, R. A., Moisan, L. J., Medina-Escobar, N., Neu, C., Cabral, A., and Parker, J. E. 2005. Arabidopsis SENESCENCEASSOCIATED GENE101 stabilizes and signals within an ENHANCED DISEASE SUSCEPTIBILITY1 complex in plant innate immunity. Plant Cell 17:2601-2613.

Gantner, J., Ordon, J., Kretschmer, C., Guerois, R., and Stuttmann, J. 2019. An EDS1-SAG101 complex is essential for TNL-mediated immunity in Nicotiana benthamiana. Plant Cell 31:2456-2474.

García, A. V., Blanvillain-Baufumé, S., Huibers, R. P., Wiermer, M., Li, G., Gobbato, E., Rietz, S., and Parker, J. E. 2010. Balanced nuclear and cytoplasmic activities of EDS1 are required for a complete plant innate immune response. PLoS Pathog. 6:e1000970.

Gehl, C., Kaufholdt, D., Hamisch, D., Bikker, R., Kudla, J., Mendel, R. R., and Hänsch, R. 2011. Quantitative analysis of dynamic protein-protein interactions in planta by a floated-leaf luciferase complementation imaging (FLuCI) assay using binary Gateway vectors. Plant J. 67: 542-553.

Glazebrook, J., Zook, M., Mert, F., Kagan, I., Rogers, E. E., Crute, I. R., Holub, E. B., Hammerschmidt, R., and Ausubel, F. M. 1997. Phytoalexin-deficient mutants of Arabidopsis reveal that PAD4 encodes a regulatory factor and that four $P A D$ genes contribute to downy mildew resistance. Genetics 146:381-392.

Guo, Y., Zheng, Z., La Clair, J. J., Chory, J., and Noel, J. P. 2013. Smokederived karrikin perception by the $\alpha / \beta$-hydrolase KAI 2 from Arabidopsis. Proc. Natl. Acad. Sci. U.S.A. 110:8284-8289.

Heidrich, K., Wirthmueller, L., Tasset, C., Pouzet, C., Deslandes, L., and Parker, J. E. 2011. Arabidopsis EDS1 connects pathogen effector recognition to cell compartment-specific immune responses. Science 334:1401-1404.

Jirage, D., Tootle, T. L., Reuber, T. L., Frost, L. N., Feys, B. J., Parker, J. E., Ausubel, F. M., and Glazebrook, J. 1999. Arabidopsis thaliana PAD4 encodes a lipase-like gene that is important for salicylic acid signaling. Proc. Natl. Acad. Sci. U.S.A. 96:13583-13588.

Jones, J. D., and Dangl, J. L. 2006. The plant immune system. Nature 444: 323-329.

Jones, J. D., Vance, R. E., and Dangl, J. L. 2016. Intracellular innate immune surveillance devices in plants and animals. Science 354: aaf6395.

Khan, F. I., Lan, D., Durrani, R., Huan, W., Zhao, Z., and Wang, Y. 2017. The lid domain in lipases: structural and functional determinant of enzymatic properties. Front. Bioeng. Biotechnol. 5:16.

Lapin, D., Kovacova, V., Sun, X., Dongus, J. A., Bhandari, D., von Born, P., Bautor, J., Guarneri, N., Rzemieniewski, J., Stuttmann, J., Beyer, A., and Parker, J. E. 2019. A coevolved EDS1-SAG101-NRG1 module mediates cell death signaling by TIR-domain immune receptors. Plant Cell 31: 2430-2455.

Louis, J., Gobbato, E., Mondal, H. A., Feys, B. J., Parker, J. E., and Shah, J. 2012a. Discrimination of Arabidopsis PAD4 activities in defense against green peach aphid and pathogens. Plant Physiol. 158:1860-1872.

Louis, J., Mondal, H. A., and Shah, J. 2012b. Green peach aphid infestation induces Arabidopsis PHYTOALEXIN-DEFICIENT4 expression at site of insect feeding. Plant Signal. Behav. 7:1431-1433.

Milligan, S. B., Bodeau, J., Yaghoobi, J., Kaloshian, I., Zabel, P., and Williamson, V. M. 1998. The root knot nematode resistance gene $M i$ from tomato is a member of the leucine zipper, nucleotide binding, leucine-rich repeat family of plant genes. Plant Cell 10:1307-1319.

Mindrebo, J. T., Nartey, C. M., Seto, Y., Burkart, M. D., and Noel, J. P. 2016. Unveiling the functional diversity of the alpha/beta hydrolase superfamily in the plant kingdom. Curr. Opin. Struct. Biol. 41:233-246.

Mine, A., Nobori, T., Salazar-Rondon, M. C., Winkelmüller, T. M., Anver, S., Becker, D., and Tsuda, K. 2017. An incoherent feed-forward loop mediates robustness and tunability in a plant immune network. EMBO Rep. 18:464-476.

Mondal, H. A., Louis, J., Archer, L., Patel, M., Nalam, V. J., Sarowar, S., Sivapalan, V., Root, D. D., and Shah, J. 2018. Arabidopsis ACTINDEPOLYMERIZING FACTOR3 is required for controlling aphid feeding from the phloem. Plant Physiol. 176:879-890.

Murase, K., Hirano, Y., Sun, T. P., and Hakoshima, T. 2008. Gibberellininduced DELLA recognition by the gibberellin receptor GID1. Nature 456:459-463.

Muskett, P. R., Kahn, K., Austin, M. J., Moisan, L. J., Sadanandom, A., Shirasu, K., Jones, J. D. G., and Parker, J. E. 2002. Arabidopsis RAR1 exerts rate-limiting control of $R$ gene-mediated defenses against multiple pathogens. Plant Cell 14:979-992.

Nalam, V., Louis, J., Patel, M., and Shah, J. 2018. Arabidopsis-green peach aphid interaction: Rearing the insect, no-choice and fecundity assays, and electrical penetration graph technique to study insect feeding behavior. Bio Protoc. 8:e2950.

Narusaka, M., Shirasu, K., Noutoshi, Y., Kubo, Y., Shiraishi, T., Iwabuchi, M., and Narusaka, Y. 2009. RRS1 and RPS4 provide a dual Resistancegene system against fungal and bacterial pathogens. Plant J. 60:218-226.

Parker, J. E., Holub, E. B., Frost, L. N., Falk, A., Gunn, N. D., and Daniels, M. J. 1996. Characterization of eds1, a mutation in Arabidopsis suppressing resistance to Peronospora parasitica specified by several different RPP genes. Plant Cell 8:2033-2046.

Pegadaraju, V., Knepper, C., Reese, J., and Shah, J. 2005. Premature leaf senescence modulated by the Arabidopsis PHYTOALEXIN DEFICIENT4 gene is associated with defense against the phloem-feeding green peach aphid. Plant Physiol. 139:1927-1934.

Pegadaraju, V., Louis, J., Singh, V., Reese, J. C., Bautor, J., Feys, B. J., Cook, G., Parker, J. E., and Shah, J. 2007. Phloem-based resistance to green peach aphid is controlled by Arabidopsis PHYTOALEXIN DEFICIENT4 without its signaling partner ENHANCED DISEASE SUSCEPTIBILITY1. Plant J. 52:332-341.

Rauwerdink, A., and Kazlauskas, R. J. 2015. How the same core catalytic machinery catalyzes 17 different reactions: The serine-histidine-aspartate catalytic triad of $\alpha / \beta$-hydrolase fold enzymes. ACS Catal. 5:6153-6176.

Rietz, S., Stamm, A., Malonek, S., Wagner, S., Becker, D., MedinaEscobar, N., Vlot, A. C., Feys, B. J., Niefind, K., and Parker, J. E. 2011. Different roles of Enhanced Disease Susceptibility1 (EDS1) bound to and dissociated from Phytoalexin Deficient4 (PAD4) in Arabidopsis immunity. New Phytol. 191:107-119.

Rossi, M., Goggin, F. L., Milligan, S. B., Kaloshian, I., Ullman, D. E., and Williamson, V. M. 1998. The nematode resistance gene $M i$ of tomato confers resistance against the potato aphid. Proc. Natl. Acad. Sci. U.S.A. 95:9750-9754.

Saucet, S. B., Ma, Y., Sarris, P. F., Furzer, O. J., Sohn, K. H., and Jones, J. D. 2015. Two linked pairs of Arabidopsis TNL resistance genes independently confer recognition of bacterial effector AvrRps4. Nat. Commun. 6:6338.

Shimada, A., Ueguchi-Tanaka, M., Nakatsu, T., Nakajima, M., Naoe, Y., Ohmiya, H., Kato, H., and Matsuoka, M. 2008. Structural basis for gibberellin recognition by its receptor GID1. Nature 456:520-523.

Singh, V., Louis, J., Ayre, B. G., Reese, J. C., Pegadaraju, V., and Shah, J. 2011. TREHALOSE PHOSPHATE SYNTHASE11-dependent trehalose metabolism promotes Arabidopsis thaliana defense against the phloemfeeding insect Myzus persicae. Plant J. 67:94-104.

Stuttmann, J., Peine, N., Garcia, A. V., Wagner, C., Choudhury, S. R., Wang, Y., James, G. V., Griebel, T., Alcázar, R., Tsuda, K., Schneeberger, K., and Parker, J. E. 2016. Arabidopsis thaliana DM2h (R8) within the Landsberg RPP1-like Resistance locus underlies three different cases of EDS1-conditioned autoimmunity. PLoS Genet. 12:e1005990.

van der Biezen, E. A., Freddie, C. T., Kahn, K., Parker, J. E., and Jones, J. D. 2002. Arabidopsis RPP4 is a member of the RPP5 multigene family of TIR-NB-LRR genes and confers downy mildew resistance through multiple signalling components. Plant J. 29:439-451.

Villada, E. S., González, E. G., López-Sesé, A. I., Castiel, A. F., and Gómez-Guillamón, M. L. 2009. Hypersensitive response to Aphis gossypii Glover in melon genotypes carrying the Vat gene. J. Exp. Bot. 60:3269-3277.

Voss, M., Toelzer, C., Bhandari, D. D., Parker, J. E., and Niefind, K. 2019. Arabidopsis immunity regulator EDS1 in a PAD4/SAG101-unbound form is a monomer with an inherently inactive conformation. J. Struct. Biol. 208:107390.

Wagner, S., Stuttmann, J., Rietz, S., Guerois, R., Brunstein, E., Bautor, J., Niefind, K., and Parker, J. E. 2013. Structural basis for signaling by exclusive EDS1 heteromeric complexes with SAG101 or PAD4 in plant innate immunity. Cell Host Microbe 14:619-630.

Wang, D., Zhang, L., Hu, J., Gao, D., Liu, X., and Sha, Y. 2018. Comparative genomic analysis of the Lipase 3 gene family in five plant species reveals distinct evolutionary origins. Genetica 146:179-185.

Witte, C.-P., Noël, L. D., Gielbert, J., Parker, J. E., and Romeis, T. 2004. Rapid one-step protein purification from plant material using the eightamino acid StrepII epitope. Plant Mol. Biol. 55:135-147.

Wroblewski, T., Piskurewicz, U., Tomczak, A., Ochoa, O., and Michelmore, R. W. 2007. Silencing of the major family of NBS-LRR-encoding genes in lettuce results in the loss of multiple resistance specificities. Plant J. 51:803-818.

Zhou, N., Tootle, T. L., Tsui, F., Klessig, D. F., and Glazebrook, J. 1998. PAD4 functions upstream from salicylic acid to control defense responses in Arabidopsis. Plant Cell 10:1021-1030. 